\title{
LA MILPA, SABERES Y LENGUAJE EN LAS PRÁCTICAS EDUCATIVAS DE NIÑOS Y DOCENTES TSOTSILES DE LA ZONA ESCOLAR 010 DE CHENALHO, CHIAPAS, MEXICO
}

\author{
MILPA, WISDOMS AND LANGUAGE IN EDUCATION PRACTICES \\ OF TSOTSILES CHILDREN AND TEACHERS OF SCHOOL ZONE 010 \\ FROM CHENALHO, CHIAPAS, MEXICO
}

\author{
Josefa Vázquez Pérez \\ Profesora en Educación Primaria para el \\ Medio Indígena en el Estado de Chiapas, México \\ josefavazquez31@gmail.com
}

Resumen: La presente investigación muestra los significados que los docentes del nivel de educación primaria indígena de la Zona Escolar 010, del municipio de Chenalho, Chiapas, México, atribuyen a su práctica pedagógica con niños tsotsiles desde el trabajo agrícola de la milpa. A través de la milpa y otras actividades, los niños y niñas tsotsiles construyen sus conocimientos desde la vida social, cultural y productiva, reivindicando que este pueblo tiene una pedagogía específica desde la cual construyen sus saberes y lenguaje, al mismo tiempo que se identificaron como estos saberes son retomados por el docente en la vinculación con los conocimientos disciplinares, como una oportunidad de legitimarlos dentro de los planes y programas nacionales, con vistas a generar habilidades, competencias y evaluaciones desde esta pedagogía, y no ser un subsistema que desarrolle conocimientos arbitrarios y de imposición cultural. Esta investigación se desarrolló desde el paradigma de base cualitativo, con enfoque interpretativocomprensivo, para comprender en profundidad dicho fenómeno, se optó por el método de estudio de caso, utilizando técnicas como los grupos focales y entrevistas en profundidad dirigidos a docentes y niños según criterio de selección, para otorgar la credibilidad de los hallazgos se realizó una triangulación teórica.

Palabras clave: milpa, actividades socioculturales, pedagogía, saberes, lenguaje, interculturalidad.

Abstract: This research reports the meanings that the teachers of the native' primary education level of the school zones 010 of the Municipality of Chiapas, México, attribute to their teaching practice with tsotsil children from the agricultural work "milpa", (the community harvest). Through the milpa and other activities, the tsotsil boys and girls build up their knowledges of the productive, cultural and social life, revendicating that these people have a specific pedagogy from which they construct their knowledge's and language. Also, it was detected how these knowledge's as an opportunity to legitimize them within the plans and curricula. This with the aim to generate skills, competences and evaluations from the pedagogy instead of being a subsystem developing arbitrary 
knowledge's due to a cultural imposition. This research was developed from the qualitative basis paradigm, with an interpretative-comprehensive focusing; and to understand this phenomenon in depth, addressed to both teachers and children according to the criterion for selection; and in order to guarantee the credibility of the findings a theoretical triangulation was made.

Keys words: milpa, sociocultural activities, pedagogy, knowledge, language, intercultures.

\section{INTRODUCCIÓN}

En los años veinte del siglo pasado se introdujo la educación escolarizada en regiones rurales de México de forma sistemática, pero no se trató de una educación orientada para los pueblos originarios. Por lo anterior, la Educación Intercultural Bilingüe en México, sigue siendo un tema que invita a reflexionar a todos los participantes del fenómeno educativo, sobre todo de los sujetos que desarrollan su práctica en los espacios indígenas.

En la actualidad se han diseñado programas para abatir las inconsistencias del subsistema educativo para el medio indígena, sin embargo, estas propuestas no han impactado significativamente en las escuelas, cuestionándose así, la pertinencia y la aplicabilidad de éstas.

Pese a que la educación, en el contexto de los pueblos originarios, es un derecho que jurídicamente se debe basar en el conjunto de saberes, lenguaje y de conocimientos ancestrales, aún no se le ha permitido florecer y resplandecer como tal.

En este sentido, la presente investigación propone comprender en profundidad las formas en que el docente significa su práctica pedagógica, en base a una actividad sociocultural como lo es la milpa, observando que de ella se desprenden saberes y lenguaje, que son necesarios para su vinculación con los conocimientos disciplinares.

\section{CARACTERIZACIÓN DEL CONTEXTO DE LA INVESTIGACIÓN}

La presente investigación se realizó en la región Altos, en el municipio de Chenalhó, Chiapas, México. Chiapas es una de las 32 entidades federativas que conforman a México, compuesta por 122 municipios y 9 regiones fisiográficas.

En la región Altos, se localiza mayoritariamente el grupo étnico tsotsil o los bast'i viniketik (hombres verdaderos); al igual que otras etnias se organizan con base a usos y costumbres, lo que equivale a decir que existen normas de convivencia, de socialización, de servicio comunitario, también roles y estatus, es decir, existe una forma cosmogónica del mundo natural, social y espiritual. 
La zona 010, ubicada dentro del municipio de Chenalhó actualmente presta servicio en educación primaria y preescolar a 19 comunidades indígenas del municipio. En el ámbito de educación primaria para el medio indígena, actualmente existen 19 escuelas de educación primaria, con un total de 70 docentes, y una población estudiantil de 1002 niños y de 994 niñas, en lo que corresponde al ciclo escolar 2016-2017.

\section{EL PROBLEMA}

La historia educativa de México en el medio indígena, atravesó por diversos procesos, desde aquellas políticas que separaron al indígena del estado nación hasta aquellas que permitieron la incorporación de los indígenas a la vida nacional con sus respectivas características, estos procesos fueron determinándola tal como hoy se plantea en la constitución mexicana, como una educación pública, laica, gratuita y obligatoria, bajo el nombre de Educación Intercultural Bilingüe ${ }^{1}$.

En estos intentos de atención a la realidad de los pueblos indígenas, se ha proporcionado a los centros escolares de educación básica indígena marcos ${ }^{2}$ y parámetros ${ }^{3}$ curriculares en donde se pone de manifiesto las necesidades de "enseñanza" de acuerdo a la "composición sociocultural" y a la lengua materna como objeto de estudio.

Sin embargo, estas prescripciones no han ayudado al proceso educativo pertinente a los niños indígenas, observando a que gradualmente existe un alejamiento de la cultura, de los saberes y también de la lengua materna.

Al respecto, Fernández de la Rota (2009) argumenta: "la propia cultura es un elemento impulsor de diversidad, tensión y estrategia, así entendida podemos afirmar que la cultura es el espacio semántico en que los seres humanos se construyen y representan a sí mismos y a los otros, y por tanto, a sus sociedades y a sus historias", entonces, si la cultura es una representación simbólica, cabría preguntarse: ¿Qué se está construyendo en la escuela al imponer una cultura, una lengua, al imponer planes y metodologías de enseñanza ajenas a una pedagogía cultural?

El conocer y trabajar desde los mundos simbólicos, posibilita que se establezca un dialogo intercultural, una visibilidad de la cultura y sobre todo, permite reconocer saberes implícitos que son importantes explicitarlos y articularlos con el conocimiento científico. Una pedagogía basada en la forma de aprendizaje de cada comunidad, supone entender su cosmovisión y desarrollar el proceso de metodología de aprendizaje situado.

En la pedagogía de los Tsotsiles, el saber es un saber que se manifiesta en la actividad, y que, también se expresa en un lenguaje, oral y corporal, por lo tanto actividad y lenguaje se complementan, los niños tsotsiles, hacen y hablan desde las prácticas socioculturales ${ }^{4}$. La Milpa, como una actividad sociocultural y agrícola básica y de tradición 
milenaria, es de alguna manera un objeto cultural y capital simbólico de todo indígena tsotsil y de todo mexicano, es decir es un punto de identidad.

De acuerdo a lo expuesto, esta investigación pretendió situar la actividad sociocultural de la milpa como una nueva opción educativa, donde los saberes, lenguajes y metodologías se pudiesen volver operantes al interior de la escuela y, donde se legitime el aprender y enseñar desde la pedagogía de los niños tsotsiles.

Como menciona Gasché (2009: p.29) “Para que la revaloración sociocultural se encarne en la juventud indígena (...) tiene que ser vivencial. Por esta razón, la escuela intercultural indígena debe preocuparse por integrar en su práctica las vivencias indígenas -no sólo las referencias verbales a ellas-; y por expresarlas y evaluarlas a partir de su aceptación positiva y placentera por los alumnos".

La relevancia de esta investigación por consiguiente, es presentar a la milpa como una nueva opción educativa o como recurso pedagógico y eje central de contenidos multidisciplinarios, por lo que, indagar e interpretar la perspectiva de los docentes en torno a los saberes y lenguajes que se generan en la milpa, constituye una etapa básica para apoyar los cambios que originen propuestas pedagógicas innovadoras en la pedagogía de ese pueblo.

\section{SISTEMA MILPA COMPONENTE ESENCIAL DE LA CULTURA MAYA TSOTSIL}

"La milpa maya se organiza en tres niveles de existencia: como sistema técnico, como sistema productivo y como sistema socio cultural" (Terán, 1994). La milpa concebida como sistema socio-cultural abarca a la familia, la comunidad, la organización política, la cosmovisión, las prácticas y costumbres asociadas a la convivencia y alimentación.

Cada sistema social o cultural tiene su dinámica propia, sus objetivos, su organización, pero todos aseguran, desde su razón de ser, que el sistema productivo funcione y se reproduzca.

Cabe mencionar que muchos de los valores para la convivencia en la comunidad se ven reflejados y aprehendidos dentro de este espacio, promoviendo y asegurando el "buen vivir" que para los tsotsiles le denominan el lekilkuxlejal. ${ }^{5}$

El "chomtik" o la milpa, es una actividad sociocultural y a la vez agrícola, se desarrolla (dependiendo de la altitud de cada municipio) durante los meses de diciembre y enero cuando se comienzan a seleccionar las mejores mazorcas, con la llegada de las primeras lluvias de abril y mayo se desgranan las mazorcas, durante los primeros días del mes de mayo algunos miembros de la familia o en compañía de la comunidad salen a su parcela a realizar la siembra. Los "iloles", son las personas encargadas de realizar ceremonias y rituales previos a la siembra, para pedir permiso a la tierra y protección a las semillas, de las lluvias, vientos y animales roedores a fin de que no hagan daño a la 
siembra, para ello se queman velas dentro de la milpa y en ocasiones se sacrifican a gallinas y esparcen la sangre en la tierra donde se cultivará; las personas adultas y sobre todo los hombres son los que inician con estas actividades, y detrás de ellos están los demás integrantes de la familia o comunidad.

Una vez iniciada la siembra se forman agujeros con un palo destinado y diseñado para la siembra, en los que se depositan de 4 a 5 semillas de maíz, terminada esta etapa, las mujeres cumplen el rol de alimentar a todos los que participaron, preparan gallina y tortillas hechas a mano. Después de algunos días, se siembra el frijol y la calabaza, tiempo que se procura estar vigilando que las aves o roedores no se coman las semillas; una vez crecida la milpa, los agricultores la limpian, quitándole las plantas que obstaculicen su crecimiento y le roben nutrientes. En un par de meses se obtienen los primeros jilotes; en septiembre abundan los elotes, y preparan diversos alimentos como, tamales de elote, tortillas, sopas etc. Finalmente en noviembre cuando la milpa comienza a secarse y el elote se convirtió en mazorca, se dobla y se espera que con las fuertes heladas por las mañanas y el calor intenso a medio día, se seque en su totalidad la mazorca. Este proceso culmina cíclicamente en diciembre cuando toda la familia va a recoger la mazorca y al mismo tiempo se eligen las mejores mazorcas y granos para la siguiente temporada.

\section{LA ACTIVIDAD DE LA MILPA COMO POTENCIAL PEDAGÓGICO}

A través de esta actividad, los niños se acostumbran a respetar, a estimar y a colaborar en el cuidado de los cultivos y de los animales, y sobre todo aprenden a leer los comportamientos.

La milpa como proceso familiar y comunitario, genera una serie de actividades culturales, conocimientos puestos en práctica o simplemente verbalizados, se desarrollan destrezas motoras, espaciales y de lenguaje, que han servido para la transmisión generacional de la cosmovisión cultural de los pueblos originarios y también para el acercamiento a nuevas formas de aprendizaje.

De alguna manera, este proceso de hacer las actividades en las comunidades, se observa de manera implícita una pedagogía indígena, que esta a su vez, se sustenta en una educación familiar y comunitaria, y que permite comparar con la educación oficial que se recibe, justamente este quiebre es el que interesa estudiar y dar respuesta al conflicto intercultural.

En este caso los saberes indígenas, "son un sistema organizado de investigación y descubrimiento, producto de una continua interacción de la gente con el medio, basada de la experiencia. Son producto de las construcciones colectivas a través de un proceso histórico en el contexto de una región específica". (Luna-Morales, 2002; Escobar-Berón, 2002, citado por Sánchez-Salinas, 2009, p. 92) 
En los procesos de acompañamiento y participación de los niños y niñas en las actividades cotidianas, los adultos no suelen emplear un discurso instruccional, simplemente desarrollan sus actividades en tanto que los niños, mediante la observación, experimentación e imitación de las vivencias en forma lúdica y reflexiva, participando en compañía de los adultos, en los escenarios reales.

Así pues, las indicaciones verbales que suelen acompañar el desarrollo del proceso práctico, son en un tipo de lenguaje que es característico y funcionalmente reconocido en los análisis de todas las lenguas indígenas: los consejos o la palabra de los mayores. Por ello, Sánchez (2009) señala que la cultura es el universo de informaciones, de valores y creencias manifiestas en símbolos, mediante un sistema de significantes que representan -símbolos de participación, étnicos, míticos, religiosos, entre otros-, dando sentido a nuestra existencia $y$, además, brindan la posibilidad de explicarnos y entender la conformación de los entes de nuestro entorno.

Finalmente, la práctica educativa debe centrarse en la lógica del grupo étnico, sin cambiar los paradigmas de la educación indígena, la relación escuela y comunidad debe verse como una relación integral y pertinente. En este sentido toda escuela debe responder verdaderamente a que la cultura local sea vivencial en las aulas y donde la lengua sea funcional para el aprendizaje e interacción del maestro con el alumno, y de esta manera generar una educación digna y horizontal.

\section{METODOLOGÍA}

El diseño metodológico de este estudio, se inscribió en el Paradigma Cualitativo, con enfoque comprensivo-interpretativo. Este estudio de caso de tipo intrínseco correspondió a uno desarrollado en la zona 10 del municipio de Chenalho, Chiapas. La muestra estructural implicó considerar una serie de criterios de selección, los informantes claves fueron 17 docentes y 10 alumnos de educación primaria indígena. El propósito, saturar el espacio simbólico para la credibilidad de dicha investigación.

Para los docentes se produjo y aplicó un grupo focal con 10 integrantes, 5 hombres y 5 mujeres. Se desarrollaron además, 7 entrevistas en profundidad a 3 hombres y 4 mujeres. Para la producción de datos de los alumnos se aplicó un grupo focal con 10 integrantes, 5 niños y 5 niñas de 9 a 12 años de edad.

\section{RESULTADOS}

En el análisis de los datos se realizó siguiendo el modelo de teorización anclado, lo que implicó la construcción de categorías a partir del análisis de los discursos. Se describen desde dos dimensiones, en la primera, se analizan datos de los docentes a partir de un grupo focal integrado por diez sujetos y de cinco entrevistas en profundidad, posteriormente se analizan los datos de un grupo focal de diez alumnos. 
El análisis de los datos producidos por profesores/as permitió la construcción de las siguientes categorías:

1. La representación de la labor docente indígena.

2. La representación de la experiencia de los docentes respecto a la milpa.

3. Propuestas para generar prácticas pedagógicas con pertinencia y valoración sociocultural.

4. Representación del valor pedagógico, social y cultural de la milpa en los espacios áulicos y extra áulicos desde la representación del docente indígena.

El análisis de datos a partir de los discursos permitió construir las siguientes categorías:

1. La asignación de roles por género en las actividades de la milpa.

2. Saberes locales adquiridos en torno a la milpa.

3. Caracterización de los procesos de enseñanza y aprendizaje.

4. La representación de las formas de acceder al aprendizaje en actividades comunitarias.

\section{REPRESENTACIONES DE LA LABOR DOCENTE}

Hace años, la figura del docente en las comunidades indígenas estaba legitimada plenamente, sin embargo hoy día el rol y posicionamiento social que se le adjudica al docente indígena ha cambiado gradualmente en muchos municipios del Estado de Chiapas, como es el caso del municipio de Chenalhó, en el que existe un alto porcentaje de profesores de escuelas bilingües, en comparación con las escuelas monolingües que son atendidas por los docentes mestizos.

“En mi experiencia como docente en este pueblo, veo que el nivel de educación indígena está muy marginado (...), en esta cabecera municipal, existen dos escuelas primarias federales monolingües o de los "kaxlanes" como se les conoce y nuestra escuela, por ello noto las grandes comparaciones que existen de parte de los padres de familia y habitantes del municipio, siempre somos vistos como la peor escuela, como aquella que solo habla en lengua indígena, y que por esta situación tenemos mala presencia, al mismo tiempo que no somos opción para la educación de sus hijos" (M5, GF)

El reconocimiento y prestigio que poseía el docente en las comunidades indígenas ha cambiado, por lo que es posible inferir que los habitantes de las comunidades le otorgan un valor importante a la labor del docente mestizo, hablantes únicamente del español, y que mayoritariamente no son indígenas, en este sentido, los habitantes observan al profesor indígena como alguien que no cuenta con las competencias necesarias para desempeñar su labor, lo que promueve retroceso social al dirigirse a los niños y población en la lengua materna de la comunidad. 
Se puede interpretar que el docente desde la perspectiva de la comunidad que promueve el aprendizaje en español, es aquel que poseía mayores competencias y habilidades, en oposición al docente que se dirige en lengua indígena. Cabría preguntarse por la sobrevaloración de la cultura mestiza por una parte, y sobre la enajenación cultural de la que están siendo parte los habitantes del municipio al preferir escuelas con docentes monolingües. Un posible cuestionamiento a la labor docente indígena y a la función de la escuela indígena en las comunidades. "Cuando llegué a esta escuela, la escuela era muy marginada, los habitantes nos decían a los maestros que solo sabíamos hablar en su idioma y que los maestros no saben" (M5, GF)

Esta escasa deslegitimación de los profesores en el medio indígena, puede responder a una situación social e histórica, al rechazo del que fueron objeto los pueblos originarios, en donde se impuso la idea de que los otros eran mejores (mestizos o kaxlanes) que las lenguas originarias era un obstáculo y que el español era válido para poder incorporarse a mejores oportunidades de vida.

Por otra parte, los docentes indígenas manifiestan que a partir de esta desvaloración hacia ellos observada en los datos, el docente también se asignan cierta responsabilidad al constructo que los padres de familia tienen sobre ellos, por la cual hacen referencia que su práctica pedagógica profesional dista mucho de la atención integral para los alumnos, tal como los profesores lo reconocen en los siguientes discursos:

La mayoría de los maestros nos conformamos con lo poquito de conocimientos que tenemos y que adquirimos en nuestra formación docente, ya que, egresando de la UPN o de las normales, ya no le dedicamos tiempo para seguir actualizándonos y modificando viejas prácticas educativas, pienso que existe cierto temor para trabajar con temas novedosos, y si no es temor, tal vez sea apatía, vergüenza. (H2, GF)

En los variados discursos de los docentes se puede inferir que la labor y la profesionalización del profesor indígena, está sujeta a muchas condicionantes, entre ellas los recursos humanos otorgados para solventar las necesidades internas de las escuela, la capacitación de calidad y con pertinencia cultural hacia los docentes y de la elaboración de materiales acordes al contexto, estas acciones no ejercidas en capacitación provocan que al docente se le deslegitime su posicionamiento sociocultural y su labor docente.

Finalmente dentro de los datos producidos por los docentes, se interpreta que el ser docente no es una decisión que se haya asumido vocacionalmente, sino más bien una necesidad y opción dentro del ramo laboral, ya que el poco interés hacia la profesionalización y actualización da cuenta de esto, por lo que nos lleva a pensar que la escuela es vista como un bien de consumo en tanto el docente accede a un pago por sus servicios. 
Por otra parte, las comunidades indígenas atraviesan por nuevas condiciones y cambios sociales como producto de la globalización que se permea en estos grupos originarios, uno de estos cambios a grandes escalas es el educativo, tal como se expresa en este discurso: "Ellos ya no quieren que a sus hijos se les hable en tsotsil, supongo que tampoco que se les enseñe desde la milpa, estar en la cabecera municipal, también implican ciertos retos." (M5, GF)

Como consecuencia, los docentes procuran que sus prácticas educativas estén lo más cercanas a los planes y programas, dejando de un lado el trabajo desde las prácticas socioculturales y de la milpa. "La milpa la sentimos como algo que no es importante enseñar, sino más bien la vemos como algo que ya está dado, que está allí, y que no se necesita buscarle más saberes" (H2, GF)

Es así que, se observa al docente como un agente reproductor de prácticas educativas hegemónicas, en donde lo que él valida como necesario e importante es aquello que esta dictado en los planes y programas de estudio y los libros de texto, además de señalar que la enseñanza se desarrolla exclusivamente dentro del salón y la escuela. Se observa que las prácticas pedagógicas desarrolladas por los docentes hacia los niños obedecen a parámetros nacionales, sin ninguna vinculación con los saberes desde la milpa.

Al igual en los discursos reflejaron que existe cierta aculturación por parte de algunos docentes, actualmente el docente ha olvidado los procesos de la milpa, muchos desconocen las temporadas lunares, se infiere que no existe una vinculación estrecha entre docente y la milpa.

Si se concibe que la escuela cumple una función vital para legitimar y perpetuar la estructura del poder y fortalecer la dominación, la escuela como una estructura estructurante debe ser selectiva tanto en lo que transmite y en cómo lo transmite, con la finalidad de garantizar en los alumnos y en la sociedad una conciencia en las formas de actuar y de pensar, por lo tanto, la relación maestro-alumnos es una relación de dominación porque conlleva un sometimiento y una arbitrariedad cultural del primero hacia el segundo, hay una violencia simbólica que impide al estudiante $y$ al docente escapar de una estructura jerarquizada en los diversos ámbitos de la vida social (Bourdieu:1970, p75).

Por ello, las prácticas educativas deberían romper con esta hegemonía. Es evidente que el niño que ingresa a la escuela trae consigo bagajes culturales y sociales que son el resultado de su proceso formativo y de socialización durante sus primeros años de vida o la socialización primaria como lo afirma Berger y Luckman. Estos conocimientos tradicionales o saberes indígenas, son un sistema organizado de investigación y descubrimiento, producto de una continúa interacción de la gente con el medio, basada de la experiencia. 
Desde este punto se puede reconocer que en las prácticas socioculturales se encuentran conocimientos valiosos posibles de incorporar en la escuela. Ante esta posibilidad, la escuela en su estructura actual se mantiene lejana o ajena a procesos de este tipo.

Pese a lo anterior, en los docentes existe un interés en cuanto al trabajo sobre actividades en la milpa, ellos reconocen que partir de estos saberes validan la lengua indígena de la población, además de que garantiza la perpetuidad de esta, por otra parte también hacen patente la importancia de trabajar con materiales que se encuentran en la región para vincularlos al conocimiento científico y de esta manera desarrollar la adaptación en las prácticas pedagógicas.

En este sentido, se demuestra la intención de los docentes en promover cambios sustanciales en su práctica, en este caso ver a la milpa como un escenario educativo, con la finalidad de retomar la cultura para el desarrollo de las actividades observándose la capacidad creativa del profesor frente a un escenario adverso como lo es el sistema indígena en el contexto chiapaneco.

Pese a todas las dificultades y carencias que el docente para el medio indígena atraviesa, que no están dentro de su alcance modificarlas, porque obedecen a cambios estructurales y de políticas educativas, el docente se le observa reflexivo y con un compromiso ético.

Se devela un aspecto importante respecto de la escuela y de la enseñanza, entendiendo que esta dicotomía, escuela y educación, no solo corresponde a un espacio físico institucional, como lo es el salón, sino más bien, hacen una vinculación estrecha entre la escuela y la comunidad, como estamentos complementarios y con responsabilidad de formación hacia los niños, de ahí, que el siguiente discurso hace mención de la importancia de los padres de familia en este proceso:

No solo el maestro es un factor influyente, sino también los padres, porque al parecer toda la responsabilidad recae en el docente, para esto los padres son un pilar muy importante para concretar una educación de calidad y de pertinencia, es decir, de los padres podemos encontrar mucha información sobre la milpa, porque nosotros ya vivimos en la ciudad y a veces no tenemos muchos conocimientos respecto a esto, entonces, ellos también los debemos involucrar, pero sería interesante, que tanto interés y compromiso asumen si tratamos de incluirlos en la escuela., porque no todo lo puede hacer el docente. Entonces profesor y padre harían un buen equipo para la toma de decisiones en torno a lo que es importante enseñar y aprender. (M5, GF)

La necesidad de modelar la práctica que los docentes para el medio indígena realizan, los lleva a buscar alternativas y sujetos de acompañamiento para una educación de calidad, por ello, sitúan a la comunidad y padres de familia como un medio para acceder al conocimiento que favorecerá procesos significativos en el salón, 
reconceptualizando que la escuela no puede ser un estamento aislado sino uno complementario dentro de la comunidad ya que los conocimientos tradicionales o saberes indígenas.

Los docentes reconocen la importancia de enseñar y aprender desde el sistema milpa, se infiere que hay una motivación importante para innovar sus actividades de enseñanza, sin embargo se observa que parte de la desmotivación es la nula capacitación sobre el tema, ellos expresan que no conocen de metodologías y de procesos didácticos que pueden ser llevados a la práctica con la finalidad de tener objetivos centrados y proyecciones importantes.

Finalmente la milpa como sistema socio-cultural abarca a la familia, la comunidad, la organización política, la cosmovisión, el conocimiento, los valores, rituales, las prácticas y costumbres asociadas a la convivencia y alimentación. Cada sistema social o cultural tiene su dinámica propia, sus objetivos, su organización, pero todos aseguran, desde su razón de ser, que el sistema productivo funcione y se reproduzca, Terán (1994).

\section{DISCURSOS DE LOS NIÑOS TSOTSILES}

A continuación, se presenta un análisis de las cuatro categorías construidas a partir de los discursos.

Al interior de las organizaciones indígenas, existen valores, normas y modos de organización que se institucionalizan con el paso del tiempo, en este caso, las comunidades tsotsiles, tienen una organización específica para realizar las actividades dentro de su grupo social, mismas que definen los roles y estatus para cada integrante de la comunidad.

Cada comunidad indígena tiene una forma única de concebir y aprender a representar el mundo indígena. De las prácticas socioculturales se desprenden la mayor cantidad de conocimientos y saberes que el niño/a a lo largo de su vida va aprendiendo, a la vez, estas prácticas ponen de manifiesto los roles y la asignación de tareas específicas para cada género, y que se perpetúan generalmente con ciertas alteraciones por el paso del tiempo y de las condicione sociales:

Las niñas ya saben lo que tienen que hacer, ellas casi siempre se quedan en casa preparando los alimentos, las tortillas, limpiando la casa, cuidando a sus gallinas, a veces van a traer leña, pero van cuando no hay hombres en la casa; y nosotros los niños, hacemos cosas que requieren más trabajo, y más fuerza. (NO2, GF)

Pero también hay muchas mujeres que saben muy bien trabajar en el campo, bueno, creo que la mayoría. (NO2, GF) 
Se observa que desde temprana edad, niños y niñas se involucran en las actividades que se desarrollan en la comunidad y familia, actividades vinculadas a la supervivencia, acceso a la alimentación, al cuidado de sí mismo y de los suyos. El niño o niña desde temprana edad ejerce su rol y posicionamiento de manera autónoma asociando la observación con la práctica. Estas formas específicas de relacionarse culturalmente, bajo la asignación de roles de acuerdo al género y edad, permiten realizar tareas vinculadas a las actividades socioculturales de la comunidad, siempre en un estricto marco de solidaridad. Los entrevistados mencionan:

Cuando era niño me correspondía ir por la leña todos los días, cargarla con un metate, también me tocaba ir por agua, a veces me tocaba pastorear a los borregos y también me tocó ir a sembrar el maíz e ir a limpiarla (...) el trabajo de las mujeres era prepararnos alimentos y cuidar de los más pequeños, mientras que nosotros los hombres nos correspondía iniciar la siembra del maíz. (H1, E)

Nosotros a nuestra edad ya empezamos a sembrar, pero generalmente sembramos frijol, nuestros papás y los hombres siembran maíz primero, y atrás de ellos sembramos frijol, creo que la edad en la que empezamos a sembrar frijol es como a los 9 o 10 años porque ya hay un poco de "Ch'ulel" (razón), pero los más pequeños de la familia van a jugar mientras nosotros trabajamos, ellos hacen pequeños mandados, como traer más semilla si no hace falta, buscar agua para nuestro pozol, los más pequeños están en la milpa, pero no hacen nada. (NO1, GF.)

Un niño indígena, provisto de todo el bagaje cultural que le rodea (lengua, indumentaria, cosmovisión), no crece aislado, sino por el contrario es parte inherente de esa realidad. Desde que nacen son parte activa de la comunidad, son seres con una participación reconocida por todos y se desenvuelven en un patrón común de socialización, mismo que se adapta a lo que la misma cultura pide de ellos.

Como lo afirma Acevedo (2000, p.137) "los indígenas no son, de ninguna manera un agregado de seres curiosos y atrasados, incapaces de hablar el español y que se empeñan en vivir en precarias condiciones, sino grupos sociales con cultura y formas de organización propias, distintas a la nacional, pero de ningún modo inferiores".

La cosmovisión de los indígenas tsotsiles es un sistema de valores que interpreta y relaciona, el mundo, la vida, las cosas y el tiempo, es además, la explicación y explicitación, y forma de dimensionar el universo y la naturaleza. La cosmovisión de los Bats'i Viniketik (o de los hombres verdaderos) es el sustento más profundo que las ancianas y ancianos crearon para explicar el origen de la vida, el origen y el desenvolvimiento holístico del universo, ya que desde esta óptica se aprecia la forma de ver, entender y vivir el sentido de la vida, así como también la espiritualidad, las ciencias, los rituales sagrados, el arte, los tejidos, la música, la lengua entre otros denotando el buen vivir, es decir el Lekilkuxlejal. 
Al mismo tiempo se observa, de manera gradual el niño y niña tsotsil aprende observando de los adultos, aprende hablando con los adultos y con sus pares, aprende también haciendo en colectividad, por lo tanto, se puede decir que dentro del sistema milpa, hay una pedagogía especifica que permite al niño acercarse al conocimiento de su mundo, pero que también le permite acercarse al conocimiento de otros mundos, en tanto este conocimiento es generador y vinculador de otras más, podemos observar que también esta pedagogía es de un carácter más instruccional.

A partir de lo expuesto, cabe la necesidad de pensar y cuestionarse qué tipo de pedagogía se desarrolla al interior de los espacios áulicos, y qué tipo de pedagogía ha implementado el modelo nacional para estas comunidades indígenas. Porque si bien, se observa que en estas actividades existe una forma única de ir construyendo conocimiento, ésta ha sido invisibilizada, sobreponiendo una pedagogía nacional, que pretende monoculturizar a toda una diversidad étnica. Por ello es menester, pensar en nuevos modelos y métodos de aprendizaje y de enseñanza, basados en una pedagogía tsotsil para este caso.

Todos los desaciertos en educación, que incluyen no solo a docentes sino a las instituciones y políticas educativas por demás, se ven cristalizados en el espacio escolar donde los inmediatos afectados son los niños.

Creemos que los maestros no les gustaría ir a trabajar en la milpa o en otras cosas (...) porque la mayoría ya vive en la ciudad. (NA1, GF)

A veces los maestros no nos quieren sacar a jugar en la cancha, o a veces cuando les decimos que queremos ir a trabajar en el campo no quieren, yo pienso que ellos no van a querer ir a nuestras milpas, también porque hay maestros que solo quieren estar en sus salones. Nosotros nos aburrimos todo el día en el salón (...) pero no podemos decir más, porque ellos son los maestros, ellos son los que saben cómo enseñarnos y lo que necesitamos aprender. (NO2, GF)

Se observa que los niños conceptualizan al docente como alguien que no se interesa y que ya no pertenece a la comunidad y al mundo indígena, además, se observa que los niños validan aún al docente por lo que dice y hace: "ellos saben lo que debemos aprender", expresa un alumno, argumento que legitima el rol docente.

La tendencia a escapar del salón de clases actitud muy generalizada en los alumnos, indica una notoria necesidad de permanecer y aprender más de su mundo de referencia, su comunidad y también del mundo exterior.

A pesar de esas debilidades, muchos niños se adaptan a la escuela considerando su importancia para acceder a oportunidades de vida. 
Estas razones hacen reformular nuestro sistema educativo, para integrar estos saberes dentro de los currículos escolares como objetos de estudio y de comprensión. Pensar, además, en la posibilidad de incluir en las actividades de las escuelas a los agentes, en este caso ancianos o adultos, que promuevan esos saberes para que se desarrollen prácticas relevantes que permitan posicionarse dentro de la escuela con eficacia y en correlación estrecha con las actividades escolares.

A nuestro juicio, la misión de toda escuela es el aumento de la capacidad de los individuos para ser sujetos. Que no solo sea un sujeto "castellanizado" o civilizado durante sus años de formación o escolarización, sino que durante toda la vida pueda desarrollarse integralmente, "Comprender al otro en su cultura, es decir comprenderlo en su esfuerzo por ligar identidad e instrumentalidad en una concepción del sujeto: tal es el sentido de una escuela del Sujeto" como lo afirma Touraine (1999).

\section{CONCLUSIONES}

Con respecto a los hallazgos sobre los significados que otorgan los docentes de educación primaria a la milpa, como proceso enraizado en la cultura, se pudo inferir que existe un vínculo cultural latente entre saberes y lengua indígena, pero que se requiere de mucha exploración e investigación de parte de la mayoría de los docentes y de la sistematización que hagan sobre ellos.

Se pudo inferir que algunos docentes atribuyen un significado mítico, y sagrado, y no enfocado a un significado educativo a la milpa, por lo que les es imposible considerarla como un espacio pedagógico y vincularla al espacio áulico. En otros docentes, en cambio, no reconocen acerca de la importancia pedagógica que pudieran tener.

Algunos docentes no disfrutan ni reconocen el mundo simbólico adscrito a la milpa, lo que nos lleva a inferir en una especie de enajenación cultural del docente que niega un universo ancestral de sentido construido.

Sin embargo a pesar de esta autopercepción estigmatizada del rol del docente respecto a la cultura, muchos profesores tienen la intención de mejorar su práctica, de generar nuevas condiciones y de situar su enseñanza con base al modelo cultural y sobre todo, situar la enseñanza desde el espacio de la milpa.

Se pudo rescatar que muchos docentes estaban orgullosos de ser maestros indígenas, y valoran a los docentes mestizos y monolingües. Consideran que tienen más cercanía y relaciones interpersonales satisfactorias con la comunidad, invierten más horas de trabajo, más distancias recorridas, más actividades que no necesariamente tienen que ver con su labor docente. Junto a esto opinan que ejercen una labor identitaria y comunitaria de revitalización de las lenguas y culturas indígenas lo que implica competencias bilingües o multilingües. 
Por otra parte, los niños tsotsiles, manifiestan que aprenden de su mundo sociocultural a partir de la observación, de la exploración y de la práctica. Se observó que en las comunidades indígenas un niño a temprana de edad se hace autónomo, desplazándose de un lugar a otro, lo que implica ir reconociendo su espacio natural y territorial.

Los niños reconocen que en la milpa se desarrollan muchos procesos de aprendizaje, de socialización, sin embargo los niños quieren aplicar un bilingüismo, señalan que requieren aprender desde un conocimiento disciplinar el proceso de la milpa, ya que no les bastaría poseer un conocimiento ancestral sobre el crecimiento de la milpa, desearían saber qué pasa bajo la tierra, quieren ser bilingües funcionales, en español y en lengua indígena.

Por estas razones, el valor pedagógico que el docente debería asignarle a la milpa y a otros espacios que todavía conservan prácticas productivas agrícolas como la siembra del maíz, puede contribuir a la valoración y revaloración de los significados propios de dichas comunidades tsotsiles y contribuir a reinvindicar la labor docente indígena frente a estos saberes propios, acercándose a la figura del docente constructivista y abierto a conocer nuevas posibilidades de enseñanza y nuevos espacios pedagógicos de aprendizaje.

Considerando la perspectiva de los profesionales expresada en una pedagogía bilingüe, la escuela debería tomar en cuenta la herencia cultural de cada pueblo y fomentar una pedagogía que facilite el desarrollo del niño a partir y dentro de su universo cultural y lingüístico local, y en oposición a una pedagogía culturalmente asimiladora que privilegia y valora los contenidos escolares nacionales ajenos al universo local.

Finalmente sin desconocer la presencia de la cultura en toda la actividad humana, en la educación forma, que se realiza al interior de los salones, se requiere enfatizar la importancia de que los saberes locales que todavía se preservan en estas comunidades tsotsiles, logren un espacio articulador y significativo en las actividades locales, como una oportunidad de conectar conocimientos en torno a procesos sistémicos de aprendizaje significativos para legitimarlos dentro de los planes y programas nacionales, y así generar habilidades y competencias desde esta pedagogía, que deje de ser un subsistema que desarrolla conocimientos arbitrarios y de imposición cultural. 


\section{NOTAS}

1. La educación Intercultural Bilingüe se inscribe en un modelo de bilingüismo que es de mantenimiento, revitalización y desarrollo de las lenguas indígenas. Privilegia la situación sociolingüística de la región y el derecho a ser educado en la lengua materna. La educación intercultural bilingüe es para poblaciones indígenas nacionales. En el año 2000 destaca el Plan de Desarrollo 2001-2006 que en lo referente a educación indígena instituye la Educación Intercultural Bilingüe (EIB) y más tarde se crea la Coordinación de Educación Intercultural Bilingüe (CEIB).

2. La diversidad y el multilingüismo obligaron al sistema educativo mexicano a crear Marcos Curriculares y, con la finalidad de que los contenidos en el Plan y Programa de estudio se vincularan con los propios del acervo cultural de los pueblos originarios y de las experiencias de los migrantes que atiende la Educación Básica. Dichos marcos pretenden dar muestra de la diversidad del país, reconociendo sus conocimientos ancestrales y actuales, propiciando el acceso a los conocimientos científicos e instrumentales provenientes del mundo en que nos movemos, al comparar y vincular las relaciones existentes entre los saberes locales como aprendizajes esperados y los aprendizajes esperados de los programas de estudio (PPE: 2011).

3. Los parámetros curriculares, fueron creados para establecer un espacio curricular para que los alumnos estudien, analicen y reflexionen sobre su lengua nativa, a partir de la apropiación de las prácticas sociales del lenguaje, orales y escritas, en los diversos ámbitos de la vida social, así como cumplir con el mandato constitucional sobre los derechos culturales y lingüísticos de los pueblos indígenas. (PPE:2011).

4. Me refiero a prácticas socioculturales, a todas aquellas actividades que desde tiempos milenarios se vienen practicando en las comunidades indígenas, algunas vistas como actividades económicas, familiares, agrícolas o de ocio, y que dentro de cada uno van insertos un cúmulo de conocimientos, lenguas y cosmovisiones. Ya que en cada uno de ellas existe una trasferencia de conocimientos vía aprendizaje por imitación o bien aprendizaje intencionado.

5. El"lekilkuxlejal" es una palabra en tsotsil, que significa "la buena vida" en palabras del lingüista Paoli (2003) menciona que supone la integración perfecta entre sociedad y naturaleza, esta buena vida existió, se ha degradado peor no se ha extinguido y es posible recuperarlo, no solo pertenece a este mundo, también más allá. 


\section{REFERENCIAS BIBLIOGRAFICAS}

Acevedo, M.L. (2000) Los niños indígenas. En: Identidad étnica y educación indígena. Antología de Ia LEP Y LEPMI 90. México: UPN.

Bourdieu, P. y Waquant, L. (1970) Respuestas. por una antropología reflexiva. Edit. Grijalbo.

Fernández de la Rota, M. (1988) Antropología social y semántica. Madrid: Consejo de Cultura Gallega.

Gasche, J. (2009) De hablar de la educación intercultural a hacerla. Colombia: Mundo Amazónico.

Paoli, A. (2003) Educación, autonomía y Lekil Kuxlejal. Aproximación sociolingüística de los Tzeltales. México: UAM-X.

Sánchez-Salinas, S. (2009) Conocimiento tradicional del aprovechamiento y consumo de insectos en la cabecera municipal de Simojovel de Allende. Tesis de Maestría del Colegio de la Frontera Sur, Chiapas, México. ECOSUR.

Terán, S. (1994) Milpa, biodiversidad y diversidad cultural. Yucatán: Estado de México.

Touraine, A. (1999) ¿'Podremos vivir juntos? Madrid: PPC. 